\title{
The Modular Ocean Data Assimilation System
}

Daniel N. Fox, Charlie N. Barron

Naval Research Laboratory. Stennis Space Center, Mississippi USA

\author{
Michael R. Carnes, Martin Booda \\ Naval Oceanographic Office. Stennis Space Center, Mississippi USA
}

This article has been published in Oceanography, Volume 15 , Number 1 , a quarterly journal of The Oceanography Society. Copyright 2001 by The Oceanography Society. All rights reserved. Reproduction of any portion of this article by photocopy machine, reposting, or other means without prior authorization of The Oceanography Society is strictly prohibited. Send all correspondence to: info@tos.org, or 5912 LeMay Road, Rockville, MD 20851-2326, USA.

\section{Germana Peggion University of Southern Mississippi - Stennis Space Center, Mississippi USA}

\author{
John Van Gurley \\ U.S. Navy, Cruiser Destroyer Group 2·Norfolk, Virginia USA
}

The modular ocean data assimilation system (MODAS) was designed to meet the U.S. Navy's need to produce rapid estimates of present and near-term ocean conditions, often in situations where little or no in situ data are available.

One of the problems facing oceanographers is the scarcity of in situ measurements to use in estimating the temperature and salinity structure of the ocean. Traditionally, we have resorted to climatologies derived from all the available historical data (Teague et al., 1990), or performed our own measurements using instruments such as expendable bathythermographs, which can be quite expensive and time-consuming. Such measurements may also be impractical in the case of mission planning or emergencies. In Navy mission execution, operators typically have access to in situ point measurements but have not had a means to fuse these data into a single picture of the ocean environment, or a means to blend these data with climatologies to fill in the data voids. Fortunately, there are several satellites that make routine measurements of sea surface temperature and height anomalies from space. These measurements can reveal information about the locations of surface features such as fronts and eddies, as well as subsurface features that can significantly affect acoustic propagation (Carnes and Mitchell, 1990; Carnes et al., 1994). The combination of in situ measurements and remotely sensed temperatures and heights forms a complex sampling pattern. The goal of MODAS is to combine these disparate data types and irregular sampling patterns to form a single integrated analysis of temperature and salinity on a regular grid.

\section{Data Distribution}

A summary of the typical distribution of data available over the northwest Atlantic is displayed in
Figure 1. The left panel shows the "ground tracks" where three orbiting satellites with onboard radar altimeters measure the sea surface height. These are the U.S. Navy's Geosat Follow-on (GFO), the European Space Agency's ERS-2, and the joint NASA-French TOPEX/POSEIDON satellites. These satellites orbit the earth in such a way as to form a repeating pattern of ground tracks. The repeat periods are approximately 17 days for GFO, 30 days for ERS2, and 10 days for TOPEX/POSEIDON. The thin lines show the complete repeating "mesh" of ground tracks from these three satellites and the thick lines show the tracks for one particular 24-hour period. The center panel shows the locations of sea-surface temperatures measured by two NOAA satellites during the same 24-hour period. The color of the dots represents the sea surface temperature estimate. In this case, purple denotes temperatures of about $0^{\circ} \mathrm{C}$ and orange denotes temperatures of about $29^{\circ} \mathrm{C}$. The right panel shows the locations where in situ measurements of the ocean were made with bathythermograph instruments dropped by ships or planes. The black dots represent expendable bathythermograph (XBT) and air-dropped XBT (AXBT) locations, where only temperature measurements are performed. The single red dot shows the location of a 'conductivitytemperature-depth' (CTD) probe, which provides both temperature and salinity. Note that the number of profiles available is so small that this panel actually displays three days of data instead of one day as in the first two panels.

\section{The Importance of Accurate Sound Speed Estimates}

MODAS consists of a collection of over 200 programs and utilities for combining irregularly sampled remote-sensed data and in situ measurements to create 


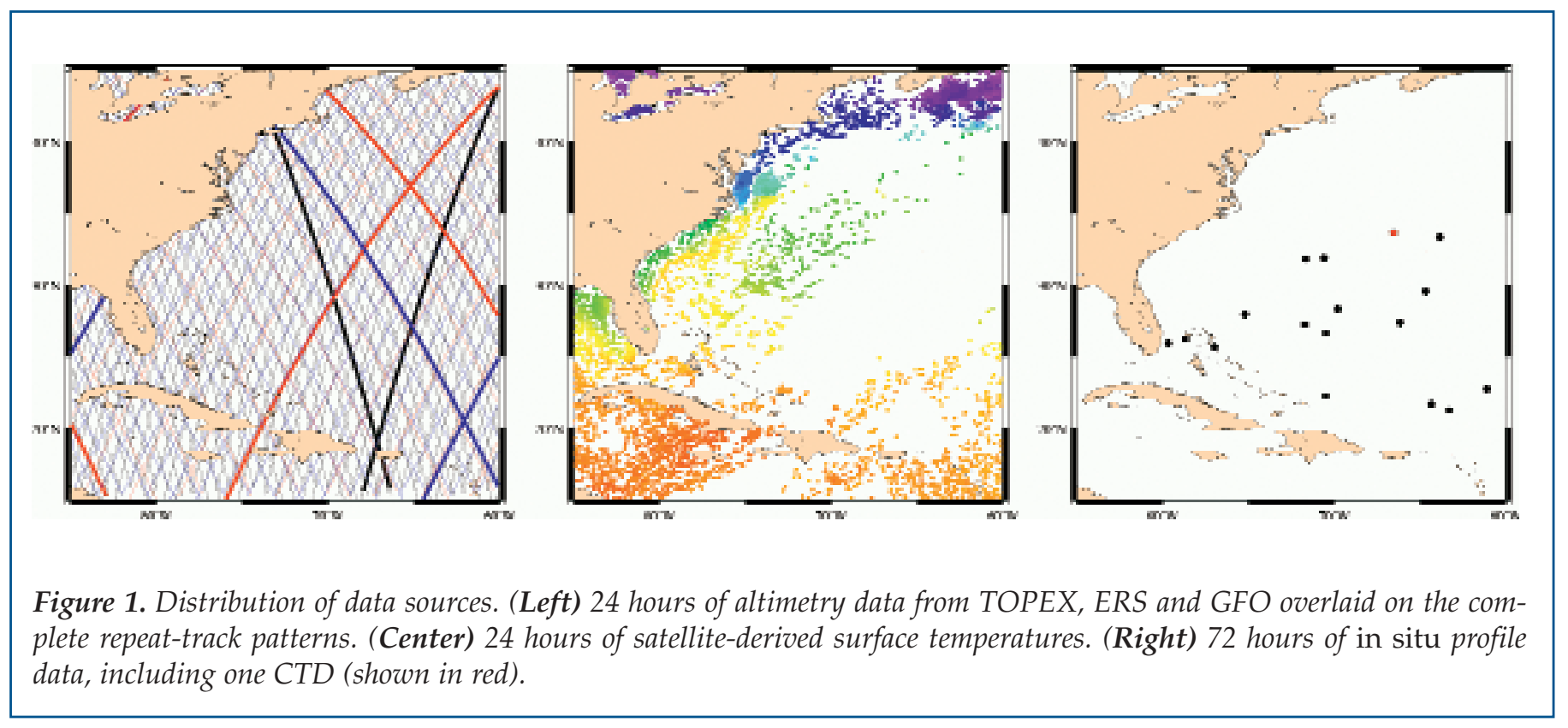

3-dimensional estimates of temperature and salinity anywhere in the ocean. Most of these are utilities for reading various data formats and providing output in various graphical and other formats that can be used in Navy systems. The temperature and salinity information produced by MODAS is assimilated into several numerical models of the ocean, providing forecasts on the evolution of these features. The temperature and salinity analyses and forecasts are typically converted into a 3-dimensional estimate of sound speed in the ocean. The sound speed structure is of particular importance since it has a direct impact on the way sound energy travels through the ocean. This is a critical issue for the Navy since it employs sound energy as the primary method of detecting and locating submarines and mines. In "active" sonar systems, a ship, plane, or submarine will use sonar to create a pulse of sound in the water and will then listen for echoes bouncing off the target submarine or mine. In "passive" sonar systems, the searcher will listen for the weak acoustic signals generated by the target submarine itself. In either case, the structure of the sound speed environment radically affects the propagation path and mode of the sound energy. Therefore knowledge of the sound speed structure is required to correctly tune the sonar systems to the environment and to choose the appropriate set of tactics to locate the target.

Variations in sound speed refract the acoustic energy and can focus it much as lenses will do with light. These variations occur in both the horizontal and vertical planes due to both dynamic oceanographic processes and hydrostatic effects due to depth. A submarine can take advantage of these variations by positioning itself to be "acoustically invisible" to ships and planes that may be searching for it. On the other hand, if those ships and planes know the sound speed structure in an area, they can use that information to improve their chances of detection. Figure 2 illustrates this situation in the vicinity of the Gulf Stream off the east coast of the United States and compares the results derived from climatology (top row) to those derived from MODAS (bottom row), using remotely sensed altimetry and temperature measurements to improve the accuracy of the sound speed. Reading from left to right, the sub-panels show sample ray paths taken by acoustic energy generated at a particular depth, and a horizontal display of "transmission loss". Sound propagation is strongly influenced by the gradient of the sound speed profile versus depth. Small changes in this gradient can have quite a significant impact, as illustrated in the right-most panels of this figure. The ultimate goal is the production of the most accurate sound speed environment possible, using whatever information is available.

MODAS approaches this problem by providing an ocean climatology, a means of converting remotesensed data into dynamic corrections to this climatology, and a procedure for incorporating in situ data, which are described in the remainder of this article. In this way, an increasingly accurate estimate of the temperature and salinity structure of the ocean is produced depending on the availability and accuracy of remotesensed and in situ measurements. Lacking any measurements, the result is simply a climatological estimate of the structure, which will lack any specific feature details such as fronts and eddies. If remote sensing is available in the form of altimetry and/or ocean surface temperature, a more accurate depiction is provided, which includes the front and eddy features detected in the remotely sensed measurements. Finally, if in situ 
measurements are available they are incorporated to add further details. These estimates of temperature and salinity can then be assimilated into global, region$\mathrm{al}$, and local numerical ocean models to provide nearterm forecasts.

\section{A "Dynamic" Climatology}

The U.S. Navy's "Master Ocean Observation Database" (MOODS) is an archive of 100 years of temperature and salinity profile measurements distributed over the world's oceans, comparable to the database maintained by the NOAA National Ocean Data Center (NODC). MODAS used these historical profiles to derive a bi-monthly, gridded climatology of temperature and salinity (Fox et al., 2002), similar to the NOAA ("Levitus") climatology and the U.S. Navy's Generalized Digital Environmental Model (GDEM; Teague et al., 1990). In addition, MODAS contains a series of regression coefficients that relate the subsurface temperature to the profile's steric height anomaly and surface temperature measurement, and also regressions derived from the historical database of profiles to estimate salinity from temperature. Thus, given a height and temperature measurement obtained from satellites, MODAS not only provides the climatological profile of temperature but also the departure of the subsurface temperature from climatology that can be estimated from surface properties, and a compatible estimate of the salinity. Typically, between $50 \%$ to $90 \%$ of the ocean's temperature and salinity variability can be estimated in this manner, even in shallow water.

Figure 3 illustrates how remotely sensed height and temperature are translated into an estimate of the subsurface temperature profile. The black line represents the climatological estimate of temperature. In this example, the satellites measured a surface temperature that was $1.5^{\circ} \mathrm{C}$ warmer than climatology. If that is the only information we have, the synthetic profile regressions simply "warm up" the whole profile (shown in red), matching the measured value at the surface-the actual correction as a function of depth having been derived from the archive of all nearby profiles. Based on the processed satellite altimetry, the steric anomaly was estimated to be $-10 \mathrm{~cm}$. That is, the height was 10 $\mathrm{cm}$ lower than the mean height calculated from climatology. This implies that the integrated water column actually has a lower temperature than climatology. The green line shows the profile that is estimated using only

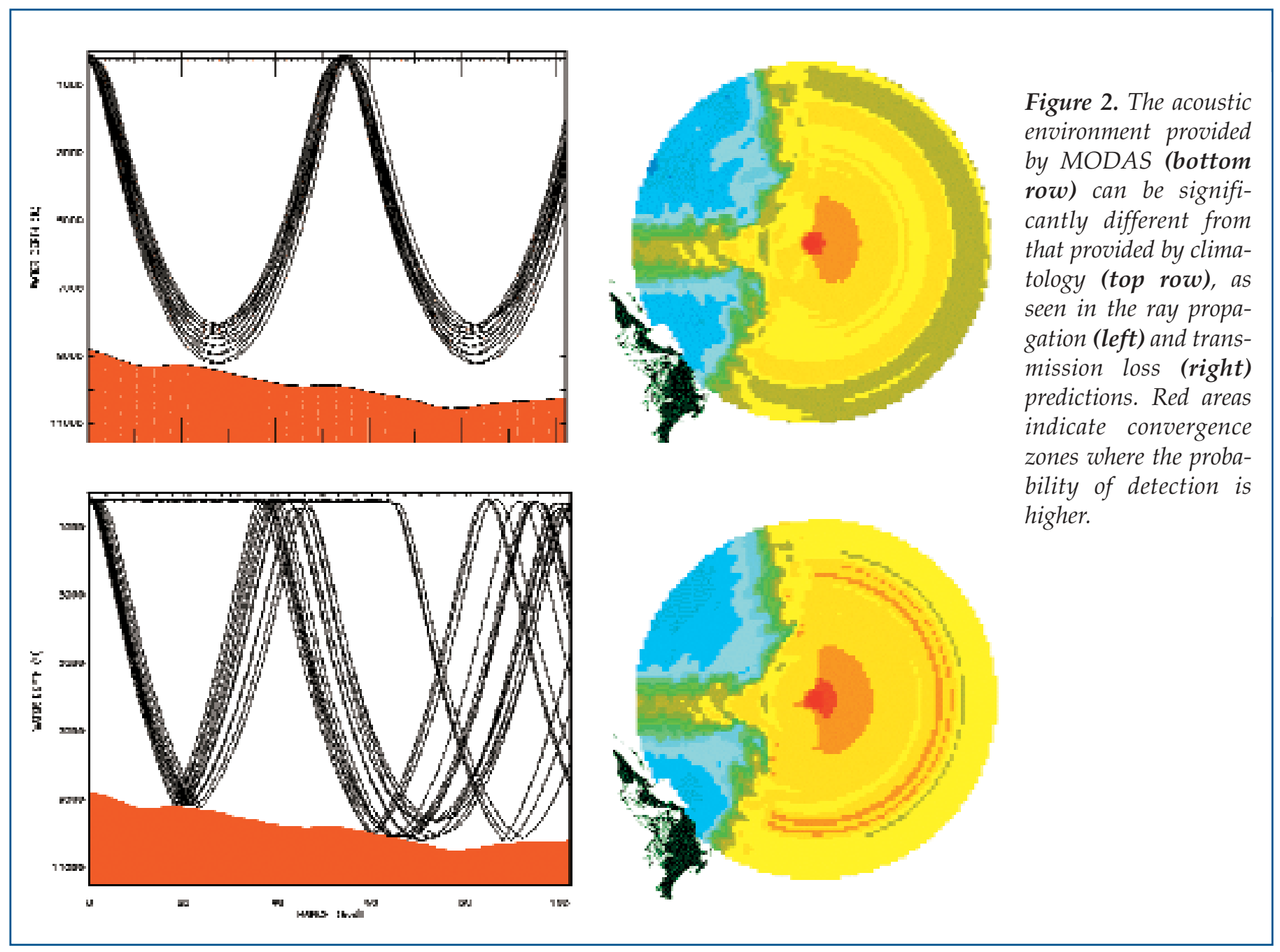




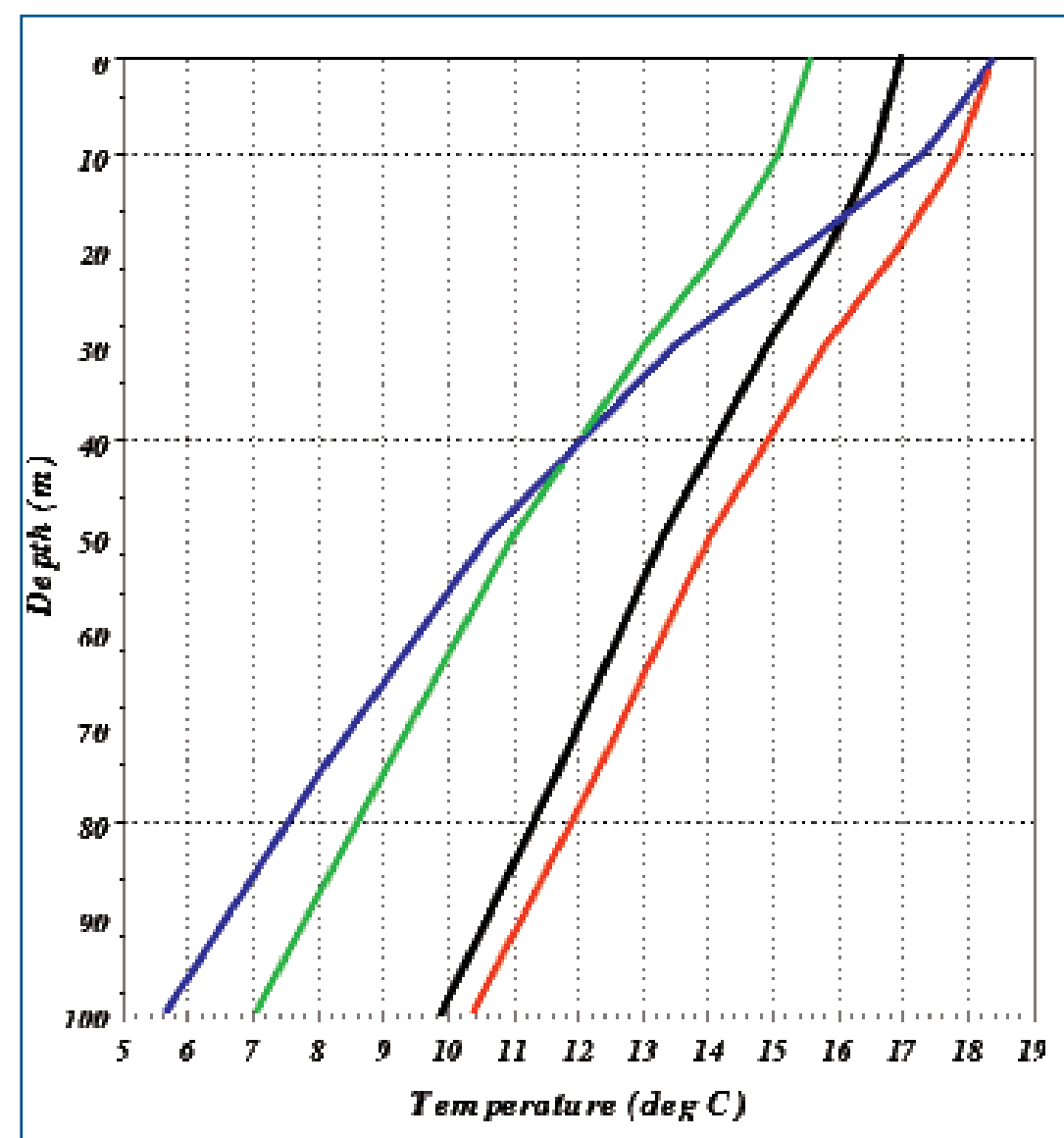

Figure 3. MODAS synthetic profiles are based on regressions derived from 100 years of in situ data. The black line represents climatology. The red line shows the profile estimated using only the remote-sensed surface temperature (which was warmer than climatology). The green line shows the profile generated using only the remote-sensed height anomaly (which indicated that the integrated water column was colder than climatology). The blue line shows the result of using both.

many years of altimetry. The details of this process are provided in Jacobs et al. in this issue. Once these 2-D temperature and height anomaly fields are produced, the synthetic profile regressions are employed to generate complete 3-D estimates of temperature and salinity globally. In Figure 4, an example of the vertical structure estimated from remote sensing is shown and compared to an in situ AXBT survey. The left panel shows a vertical section extracted from climatology, and the center panel shows the section estimated using the remotely sensed data and the MODAS synthetic profile algorithms. Note how well this estimate compares to the actual survey shown in the right panel. In this case, the altimetry has revealed the presence of a subsurface cold-core eddy that was not seen in the remote-sensed temperature data.

\section{Incorporation of In Situ Measurements}

MODAS was designed not only to provide an improved temperature, salinity, and sound speed estimate from remote sensed measurements, but also to exploit in situ measurements to further improve the analysis. The method presently employed is known as optimum interpolation (Bretherton et al., 1976), an efficient and commonly used technique for combining an initial background field and measured data (both of which are assumed to have errors) using a model of how nearby data are correlated. Often, the correlation is assumed to have a simple exponential or Gaussian form that includes a local length

the remotely sensed heights for this example. If we use both the altimetry (which says the overall profile is colder than normal) and the remote sensed temperatures (which say that the surface is warmer than normal), the regressions provide the final estimate of the profile, shown in blue. The specific correction as a function of depth depends on the location and the time of year. MODAS thus includes a gridded global database of regression coefficients derived from all the historical profiles that are sufficiently "near" each of these grid nodes.

In order to produce a complete 3-dimensional volume of temperature and salinity, the raw scattered Multi-Channel Sea Surface Temperature (MCSST) measurements and along-track height estimates are interpolated to form 2-D grids of surface temperature and height anomaly using optimum interpolation, described in more detail in the next section. In the case of the altimeter data, a complex covariance function is employed that includes spatially varying length and time scales as well as propagation terms derived from and time scale that describes the quantity being estimated. Given an initial estimate of the field (called a "first guess") and its error, a set of data points with known or estimated errors, and knowledge about the historical length and time scales of variability in the area, the optimum interpolation technique provides a set of weights to apply to the data in order to combine all this information consistently. The result is a new estimate of the field, including an estimate of its accuracy.

Optimum interpolation is used to combine in situ measurements of temperature and (where available) salinity profiles with the 3-D volumes of temperature and salinity estimated from the remote sensed data described in the previous section. In the case of temperature, for example, all the recently observed temperature profiles in the area are subtracted from the first guess field to produce a set of profiles of temperature anomalies. At each point in the grid and at each desired depth, a weighted estimate of these anomalies is computed using optimum interpolation. The result is a complete 3-D field of anomalies that is then added 

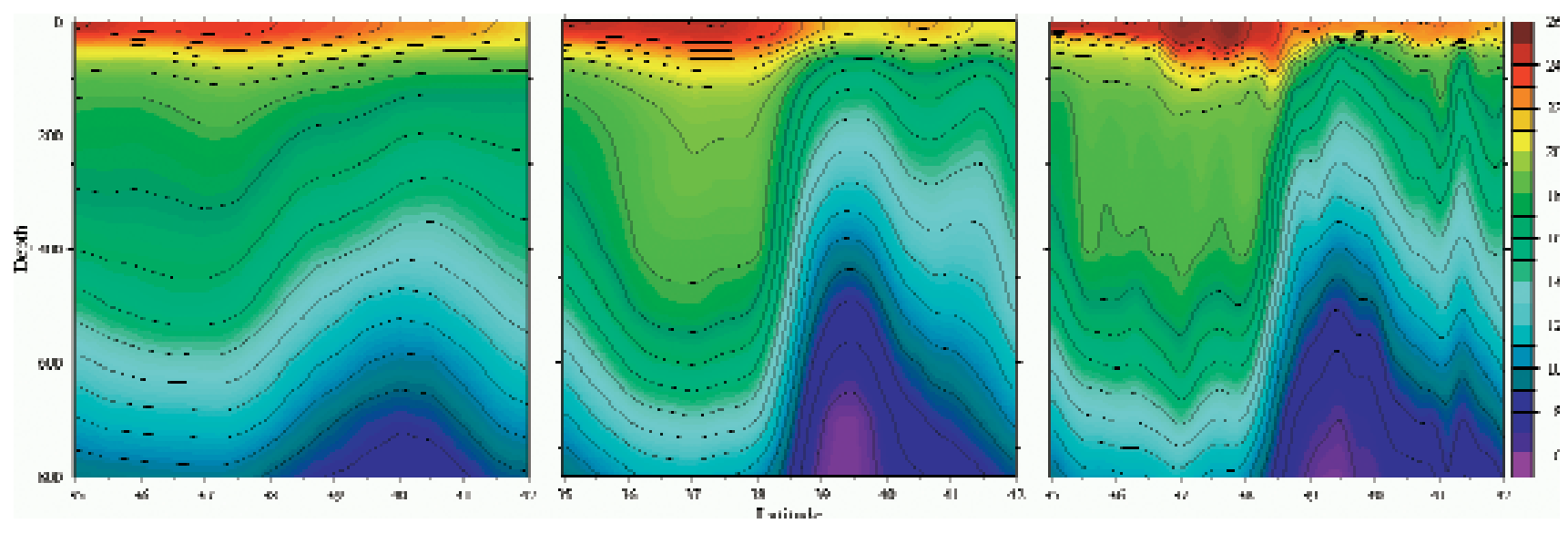

Figure 4. The MODAS "dynamic climatology" uses remotely sensed altimetry and temperatures to provide a more accurate subsurface structure than traditional climatologies. (Left) vertical cross section of climatological temperature. (Center) the MODAS dynamic climatology derived using only remotely sensed data. (Right) section measured by a dense profile survey.

back to the first guess field to form the final analysis. The 3-D temperature estimate is then converted to salinity using temperature-salinity regression relationships stored in the MODAS regression database, and the process is repeated to combine the available salinity profile measurements with this first guess salinity volume.

Before the optimum interpolation process is applied to the temperature and salinity profiles, they are passed through a quality-control (QC) stage that attempts to detect and remove bad observations. Profile measurements are initially compared to climatology and excessive differences are flagged as being possibly bad. A "mini-optimum-interpolation" is also done whereby each profile is estimated using only other nearby profiles to check for consistency. A set of profiles that are anomalous but which compare well with their neighbors may represent a situation such as an El Niño, where an entire large area of the ocean might be anomalously warm relative to climatology.

\section{Short-Term Ocean Forecasts}

Temperature and salinity estimates produced by MODAS are assimilated into a $1 / 8^{\circ}$ resolution global thermodynamic ocean model (see Rhodes et al., this issue). MODAS itself also includes a module called NRLPOM that is based on the Princeton Ocean Model (Mellor, 1992) that can be used to provide relatively high-resolution short-term forecasts, including the effects of tidal forcing, and which can use winds from high-resolution atmospheric models such as COAMPS (see Hodur et al., this issue). The NRLPOM module includes several initialization and boundary condition options selectable by the user for specific conditions.
Initialization options include:

- a "cold start" mode where the MODAS temperature and salinity fields are used but not the geostrophically estimated currents

- a "warm start" mode which does use the MODAS-estimated currents or currents extracted from a larger numerical ocean model domain

- a "diagnostic" mode where the POM model is "run in place" for a day or two holding the MODAS T and S fixed but allowing the model to spin up its own current field

Boundary condition options include:

- MODAS-derived transports along the boundaries

- Transports obtained from other regional or global models

In addition the boundaries can be forced with tidal heights, such as those obtained from the global FES95.2 ("Grenoble") tide model (Le Provost et al., 1994), so that the NRLPOM model can include the influence of tides in the area.

The model is forced using operational winds from either the global NOGAPS atmospheric model (see Rosmond et al., this issue) or regional and local COAMPS models (see Hodur et al., this issue).

Figure 5 shows an example of a triple-nest off the coast of southern California. Transports were extracted from the $1 /{ }^{1}{ }^{\circ}$ resolution global NCOM model (see Rhodes et al., this issue) to drive a regional southern California model (at $6 \mathrm{~km}$ resolution) which then provides boundary conditions for the small high-resolution $(1.5 \mathrm{~km})$ model as shown. 

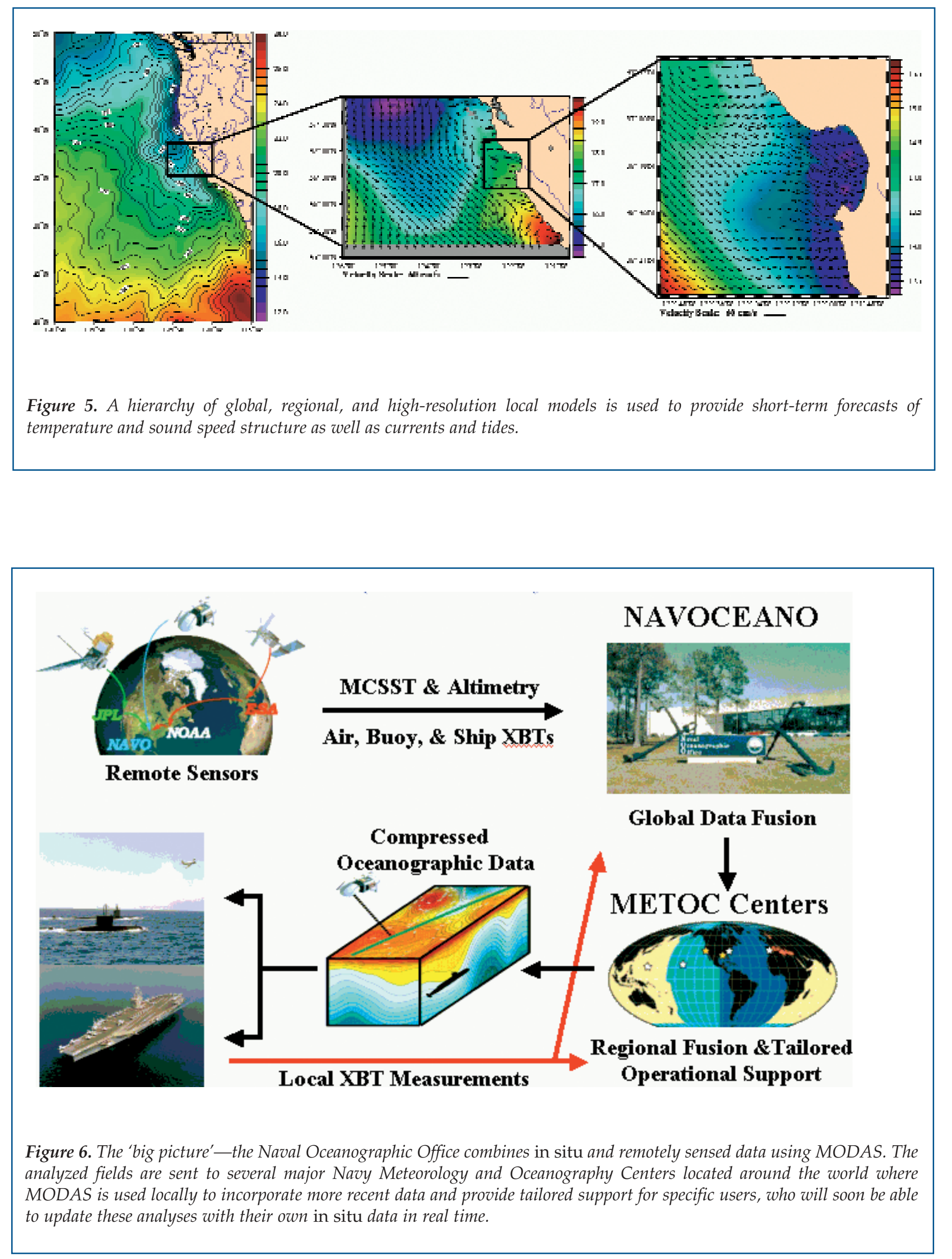
Putting It All Together

The Navy has two large-scale computing facilities for producing operational atmospheric and oceanographic analyses and forecasts, the atmosphere being primarily handled by the Fleet Numerical Meteorology and Oceanography Center (FNMOC) in Monterey California, and the ocean being primarily handled by the Naval Oceanographic Office (NAVOCEANO) at Stennis Space Center, Mississippi. In addition, several regional meteorology and oceanography centers are scattered around the world with responsibilities for specific regions. Focusing on the oceanographic analyses and forecasts, the Naval Oceanographic Office processes the global altimetry and MCSST data using a 2-D version of MODAS (see Jacobs et al., this issue). The resulting 2-D grids of height anomalies and temperatures are converted into 3-D grids of temperature and salinity using the MODAS dynamic climatology described above. All the available in situ measurements of temperature and salinity are then blended into this "first guess" using optimum interpolation to produce about 30 regional analyses which are transmitted to the regional meteorology and oceanography centers around the world once each day. The regional centers then use these fields as first guesses to further assimilate more recent temperature and salinity profiles, which are then sent to ships and submarines at sea. In addition to the automated data quality control provided by MODAS, operators at the regional centers have the option of manually viewing and editing the in situ profile measurements. The currents provided by the relocatable POM model component are used by a "drift" model which estimates how objects at the surface migrate over time; useful in search and rescue operations, for example.

Both at NAVOCEANO and the regional centers, a major subset of the modules in MODAS were designed to deal with the numerous data formats in use by Navy systems so that it can ingest as much data as possible and produce output in formats that can be directly used in these other systems. Figure 6 illustrates the flow of temperature and salinity profile data from the field back to NAVOCEANO where it is combined with the remote sensed data and sent back out as analyzed regional fields for local use. One of the critical "choke points" in the system is the relatively low bandwidth available for communications to some ships and submarines, for which a method of compressing the oceanographic data using wavelets (Fowler and Fox, 2001) was developed.

\section{Future Directions}

MODAS will soon be deployed directly onboard ships and submarines so that temperature and salinity data acquired locally can be assimilated immediately, rather than waiting for the data to be transmitted back to a regional center or the Naval Oceanographic Office to be incorporated into their analyses. In addition, sub- marines and autonomous underwater vehicles can provide temperature and salinity measurements along their "flight paths" and MODAS is being upgraded to support assimilation of these data virtually in real time, providing the submariner with a continuously updated three-dimensional depiction of the local sound speed environment.

\section{Acknowledgements}

The authors wish to express their gratitude to the many members of the Navy oceanography community who have contributed invaluable suggestions over the years that have directly lead to numerous improvements to the system. This work has been funded by the Office of Naval Research (program element 0602435N) and the Space and Naval Warfare Systems Command (program element $0603207 \mathrm{~N}$ ). This paper, NRL contribution NRL/JA/7320/01/0027, is approved for public release, distribution unlimited.

\section{References}

Bretherton, F.P., R.E. Davis and C.B. Fandry, 1976: A technique for objective analysis and design of oceanographic experiments applied to MODE-73, Deep Sea Res., 23, 559-582.

Carnes, M.R. and J.L. Mitchell, 1990: Synthetic temperature profiles from Geosat altimetry: comparison with air dropped expendable bathythermograph profiles. J. Geophys. Res., 95(C10): 17979-17992.

Carnes, M.R., W.J. Teague and J.L. Mitchell, 1994: Inference of subsurface thermohaline structure from fields measurable by satellite. J. Atmos and Oc. Tech., 11(2).

Fowler, J.E. and D.N. Fox, 2001: Embedded WaveletBased Coding of Three-Dimensional Oceanographic Images with Land Masses. IEEE Transactions on Geoscience and Remote Sensing, 39(2), 284-290.

Fox, D.N., W.J. Teague, C.N. Barron and M.R. Carnes, 2002: The Modular Ocean Data Assimilation System, Journal of Atmospheric and Oceanic Technology (accepted).

Le Provost, C., M.L. Genco, F. Lyard, P. Vincent and P. Canceil, 1994: Spectroscopy of the world ocean tides form a finite element hydrodynamic model, $J$. Geophys. Res., 99, 24,777-24,798.

Mellor, G.L, 1992: User's Guide for a ThreeDimensional, Primitive Equation, Numerical Ocean Model, 35 pp., Prog. in Atmos. and Ocean. Sci., Princeton University.

Teague, W.J., M.J. Carron and P.J. Hogan, 1990: A comparison between the Generalized Digital Environmental Model and Levitus climatologies, J. Geophys. Res., 95, 7167-7183. 